\title{
Posterolateral Intertransverse Lumbar Arthrodesis in the New Zealand White Rabbit Model: The Illustration of an Alternative Surgical Approach
}

\author{
Zunariah Ba, Zamzuri Z ${ }^{b}$, Che Nor Zarida CS $^{b}$, Rosnani AJ \\ aDepartment of Basic Medical Sciences, Kulliyyah of Medicine, International Islamic University Malaysia \\ ${ }^{b}$ Department of Orthopedics, Traumatology and Rehabilitation, Kulliyyah of Medicine, International \\ Islamic University Malaysia
}

\begin{abstract}
Introduction: The New Zealand White (NZW) rabbit model is commonly used for the research of posterolateral intertransverse lumbar arthrodesis. The standard approach reported by many researchers are a muscle splitting approach through the intermuscular plane between multifidus and longissimus muscles. Methods: In this research the fusion bed was exposed by retracting the three groups of paraspinal muscles (multifidus, longissimus and iliocostalis) medially. Results: This alternative method showed good exposure of the transverse processes and intertransverse membrane with less bleeding from the muscles observed. No death due to surgical complication was observed out of twenty-four rabbits operated. Conclusion: An easy and safe method for performing posterolateral intertransverse lumbar arthrodesis was demonstrated and recommended as an alternative approach for surgery on New Zealand White rabbits.
\end{abstract}

KEYWORDS: Lumbar vertebrae, arthrodesis, surgical approach, spinal fusion, NZW rabbits.

\section{INTRODUCTION}

The New Zealand White (NZW) rabbit model is beingused extensivelyintheresearch of posterolateral intertransverse lumbar arthrodesis. Studying bone formation and its biology at intertransverse posterolateral lumbar arthrodesis requires the use of a suitable animal model. ${ }^{1-3}$ In this animal model, the standard approach reported by many studies is by muscle splitting approach. ${ }^{4-7}$ This approach was characterized by Boden et al.(1995); following which many studies reported their surgical technique for posterolateral intertransverse lumbar arthrodesis based on this approach. ${ }^{1}$ Detail descriptions of the surgical anatomy and operative technique using a similar animal model was reported by Palumbo et al.(2004) and Valdes et al. (2004) respectively. ${ }^{8,9}$ In this surgical technique, as described by Boden et al(1995), a dorsal midline skin incision was made, which was followed by two paramedian fascial incisions. Subsequently, the fusion bed was exposed via intermuscular plane

Corresponding author:

Dr Zamzuri Zakaria

Associate Professor,

Department of Orthopaedics, Traumatology \&

Rehabilitation, Kulliyyah of Medicine,

International Islamic University Malaysia,

Jalan Hospital,

25150 Kuantan, Pahang, Malaysia

Tel: +60139906744

Fax: +6095144451

Email:zamdr@yahoo.co.uk between multifidus and longissimus muscle. We had a difficulty at the early stage of the research using this approach and with details understanding the anatomy of the rabbit lumbar spine the alternative approach was developed. In contrast to the posterolateral surgical technique as described by Boden et al, in our approach, the fusion bed was exposed by retracting all the paraspinal muscles (multifidus, longissimus and iliocostalis) medially. Therefore, the aim of this study was to demonstrate an open surgical technique for the lateral approach as an alternative surgical approach for intertransverse lumbar arthrodesis in the NZW rabbit model.

\section{MATERIALS AND METHODS}

This study was approved by the Ethics Committee, Kulliyyah of Medicine, International Islamic University Malaysia. Twenty-four skeletally mature NZW rabbits underwent single level bilateral posterolateral intertransverse spinal fusion at L5 L6 level. The age of animals ranged from 10 to 19 months old with the range weight between 2.25 to $3.40 \mathrm{~kg}$. One orthopaedic surgeon performed the surgical procedures. Anesthesia was administrated by a trained laboratory technician.

\section{EXPERIMENTAL PROTOCOL \\ Anesthesia}

Preoperatively, the animals were weighed to determine the amount of medication and anesthetic drugs. The animals were induced with a combination of Ketamine (Ketapex, Australia), Tilatamine/ 
Zolazepam (Zoletil-50, France) and Xylazine (Ilium Xylazil-20, Australia). ${ }^{10}$ The mixture was given at $0.2 \mathrm{ml} / \mathrm{kg}$ body weight intramuscularly for the induction and maintained at $0.1 \mathrm{ml} / \mathrm{kg}$ intravenously during the surgery.

\section{Preparation for surgery}

The animals were put in a prone position, and the dorsal aspect of the lumbar spine was shaved. The surgical field was prepared and draped using a sterile technique. The surgeon and the assistant must wear sterile gloves, gowns, caps and face masks to avoid infection. The spinous processes of L4 to L7 were marked (Figure 1a). Preoperative antibiotic, enrofloxin $10 \mathrm{mg} / \mathrm{kg}$ (Baytril ${ }^{\circledR} 5 \%$, Germany) was given as prophylaxis prior to skin incision.

Surgical approach

A single midline skin incision measuring about $6 \mathrm{~cm}$ was made, centered over the L4 to L7 level (Figure 1b). Left and right $4 \mathrm{~cm}$ longitudinal paramedian incisions, $2 \mathrm{~cm}$ lateral to the midline were made through the lumbar fascia to expose the paraspinal muscles (Figure 1c). The lumbar fascia was retracted laterally and the lateral border of iliocostalis muscle was identified by a blunt finger dissection and the tips of the transverse processes of lumbar spines were palpated deep to this muscle. Using a periosteal elevator, the muscles were stripped from the respective transverse processes up to the base (Figure 1d). The hemostasis was secured during the procedure. These muscles were then retracted medially with a retractor exposing the whole length of transverse processes and the intertransverse ligament, which later prepared as a fusion bed (Figure 1e). Later, the wound was closed with a bioabsorbable surgical suture and flavin dressing was applied.
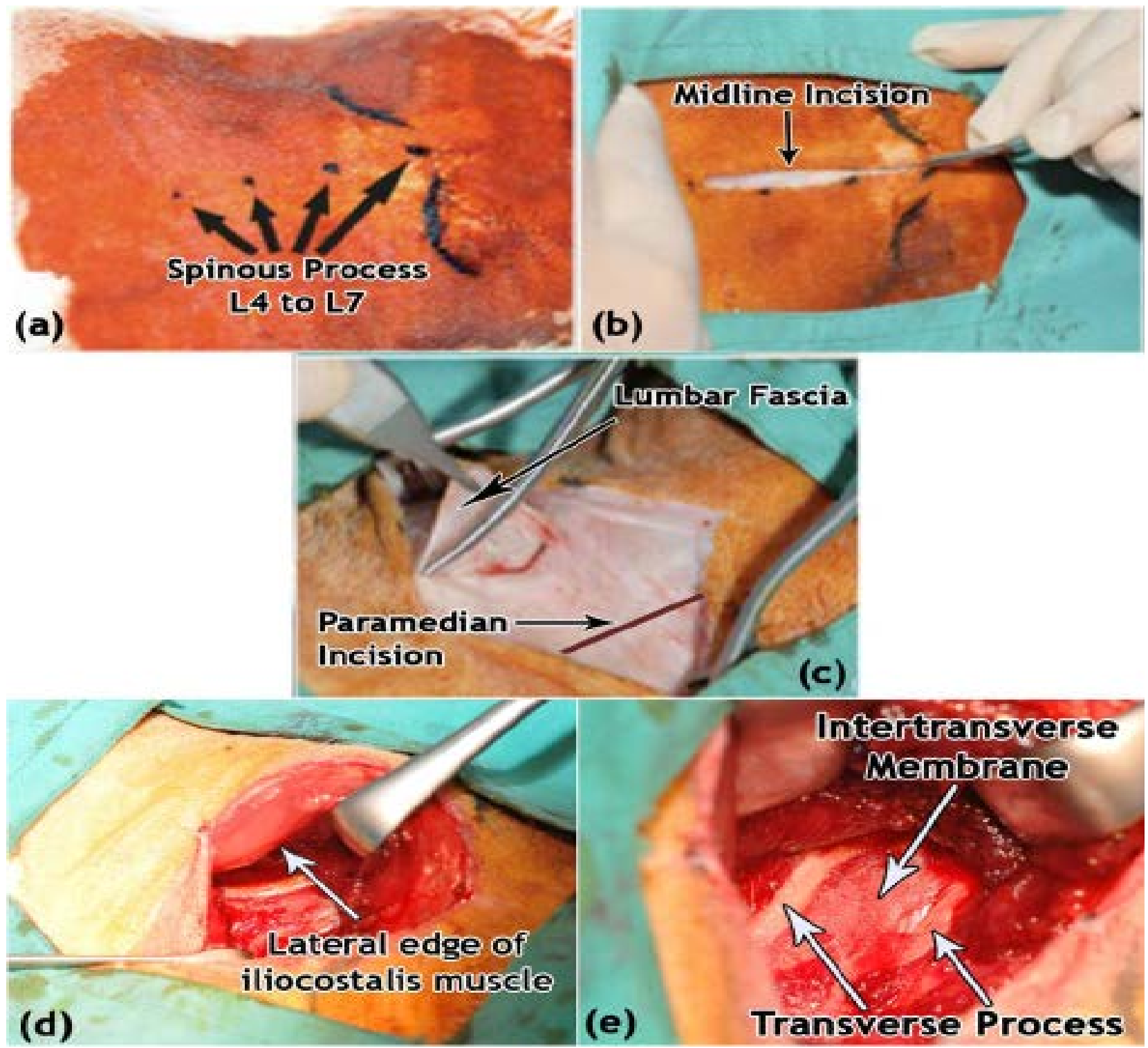

Figure 1(a). The rabbit in prone position, shaved and the spinous processes L4 to L7 were marked. (b) A single midline skin incision. (c) The line shows an imaginary paramedian fascial incision while the contralateral side shows the cut lumbodorsal fascia and the incision. (d) The paraspinal muscles were retracted medially and the relevant lumbar vertebrae were identified. (e) Exposed intertransverse membrane and transverse processes. 
Post-operative care

The animals were housed in a cage individually and accessed to food and water ad libitum. There was no postoperative restriction of activity, and no supportive orthotic devices were used. Tramadol $(5 \mathrm{mg} / \mathrm{kg})$ was given intramuscularly for the first 48 hours and followed by subcutaneous injection of Metamizole (Novasul,Australia) daily up to five-day post surgery for pain control. Intermittent subcutaneous saline was given to animals with poor oral intake to prevent dehydration. The animals were monitored twice daily for the first two days and daily until the time of sacrifice. Feeding pattern, abnormal behaviour , temperature, weight, posture, and other complications were recorded.

\section{RESULTS}

All rabbits tolerated the surgical procedure well. The operative time per animal ranged from 65 to 155 minutes. The amount of blood loss ranged from less than 10 to $45 \mathrm{ml}$. Blood loss during the surgery was mainly during harvesting the corticocancellous bone from the iliac crest and during the clearance of fusion bed from the attached muscles.
Complications noted were deep infection (4 out of 48 samples) and wrong level of fusion (3 out of 48 samples). Total exclusion rate was $15 \%$ (7 out of 48 samples). There was no intraoperative death and no evidence of nerve palsy following the surgery.

\section{DISCUSSION}

New Zealand white rabbit is an established animal model to study the basic biology of lumbar arthrodesis. Many studies described the surgical approach based on paraspinal muscle-splitting procedure as reported by Boden et al. ${ }^{1}$ In this technique, the fusion bed was approached through the intermuscular plane between the multifidus and longissimus muscles (Figure 2a). We described an alternative technique where the fusion bed was approached by elevating the iliocostalis muscle without disturbing the multifidus and longissimus muscle (Figure 2b).Although this approach has been described in video-assisted surgery, but, it is never been described in details as an open surgery in the New Zealand white rabbit model. ${ }^{11}$

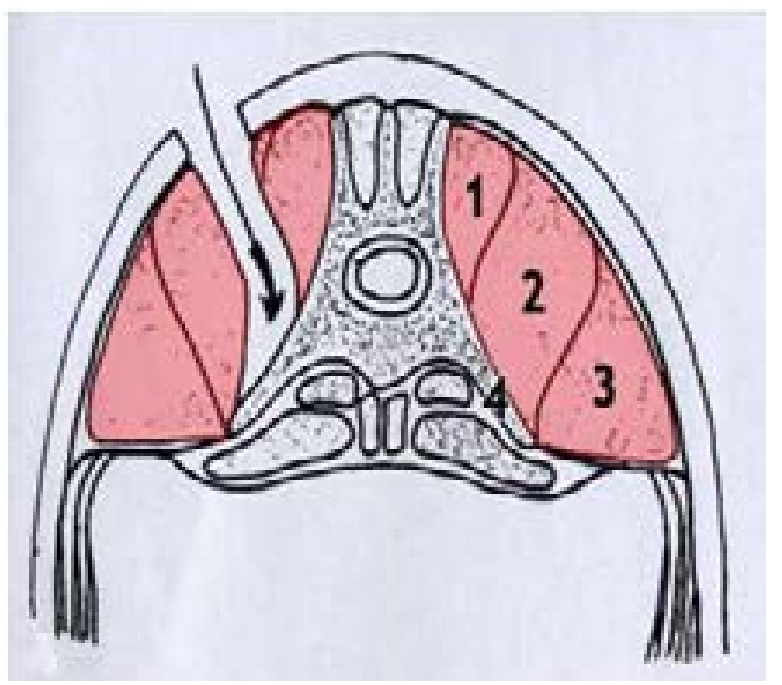

A

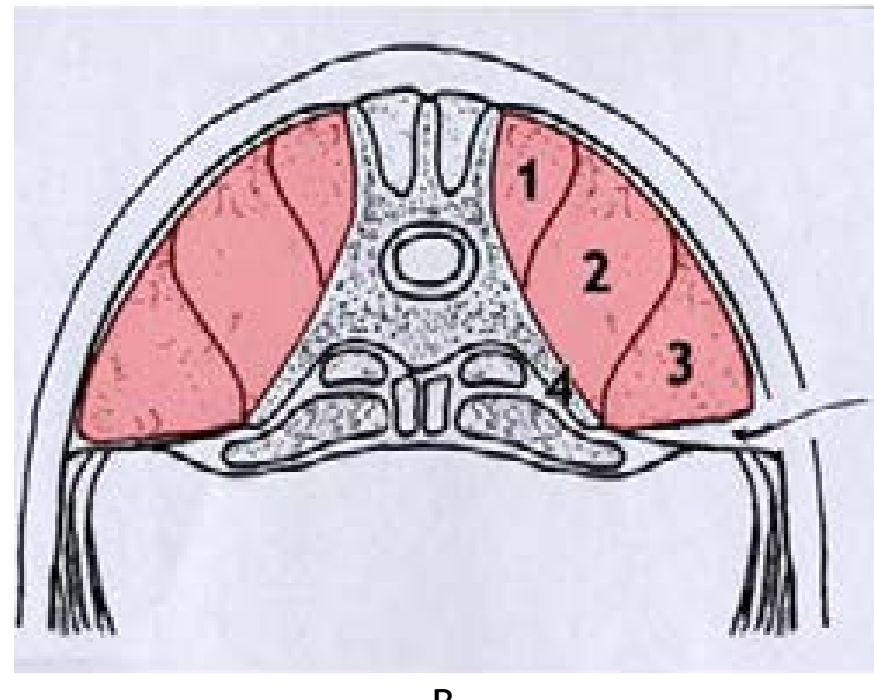

B

Figure2(A) Fusion bed was approached through the intermuscular plane between the multifidus and longissimus muscle.(B) Fusion bed was approached by identifying and elevating the iliocostalis muscle. $1=-$ multifidus muscle; 2 =longissimus muscle; $3=i$ iliocostalis muscle; $4=$ transverse process. (Modified from the video-assisted lateral intertransverse process arthrodesis: validation of a new minimally invasive lumbar spinal fusion technique in the rabbit and nonhuman primate (rhesus) models. Spine 1996; 21 (22): 2689-2697)

The important landmark for the lateral approach is the transverse processes of New Zealand white rabbit as the tip anatomically became flattened into a thin, triangular plate of cortical bone, and the length was significantly increased from L1 to L6. ${ }^{8}$ The tips were easily palpable and served as an advantage with our approach. Furthermore, the ventral inclination of the transverse processes at the lumbar spine made this approach easier by elevating the iliocostalis muscle from the lateral side to expose the fusion bed. The other advantage of this approach was less trauma to the multifidus and longissimus muscles, thus the amount of bleeding observed from the muscles was less as the intermuscular plane was not disturbed.
Therefore, we found these alternative approaches were beneficial for our study. Deep wound infection and wrong level of fusions were the main complications observed during the study. We minimized our infection with administration of antibiotics and strictly adhering to the principles of sterile surgical technique. Careful handling of the soft tissue, less operating time and efficient operative technique further reduce the rate of infection. Clinically, we checked our level of fusion by palpating and comparing the distance of interspinous space of L5-L6,L6-L7,L7-S or between the sacral processes where the L6-L7 interspinous space has the widest distance as compared to the other interspinous space. ${ }^{8}$ 
Three out of 48 samples had a wrong level of fusion, and the result was comparable with the study done by Valdes et al. ${ }^{9}$ We recommended a preliminary anteroposterior radiograph of the lumbar spine to detect anomalies and reduce the rate of wrong level of fusion.

\section{CONCLUSION}

This approach demonstrates good exposure of transverse processes with minimal damage to the surrounding muscles and resulted in less bleeding from the muscles. Using this approach, a safe and reproducible method for performing posterolateral intertransverse lumbar arthrodesis surgery on rabbits was demonstrated.

\section{ACKNOWLEDGEMENTS}

The authors would like to thank Kulliyyah (Faculty) of Medicine, International Islamic University Malaysia for providing study necessities and Ministry of Higher Education for the financial support under the Fundamental Research Grant Scheme (0207-69).

\section{REFERENCES:}

1. Boden SD, Schimandle JH, Hutton WC. An experimental lumbar intertransverse pro cess spinal fusion model. Radiographic, his tologic, and biomechanical healing charac teristics. Spine 1995; 20:412-20.

2. Curylo LJ, Johnstone B, Petersilge CA, Janicki JA, Yoo JU. Augmentation of spinal arthrodesis with autologous bone marrow in a rabbit posterolateral spine fusion model. Spine 1999;24:434-9.

3. Rao RD, Gourab K, Bagaria VB, et al. The effect of platelet-rich plasma and bone mar row on murine posterolateral lumbar spine arthrodesis with bone morphogenetic pro tein. J Bone Joint Surg Am 2009;91:1199206.

4. Magit DP, Maak T, Trioano N, et al. Healos/ recombinant human growth and differentia tion factor- 5 induces posterolateral lumbar fusion in a New Zealand white rabbit mod el. Spine 2006; 31:2180-8.

5. Martin GJ Jr, Boden SD, Titus L. Recombi nant human bone morphogenetic protein-2 overcomes the inhibitory effect of ketoro lac, a nonsteroidal anti-inflammatory drug (NSAID), on posterolateral lumbar inter transverse process spine fusion. Spine 1999; 24:2188-93.

6. Grauer JN, Patel TC, Erulkar JS, et al. 2000 Young Investigator Research Award winner. Evaluation of OP-1 as a graft substitute for intertransverse process lumbar fusion. Spine 2001;26:127-33.

7. Grauer JN, Vaccaro AR, Kato M, et al. De velopment of a New Zealand white rabbit model of spinal pseudarthrosis repair and evaluation of the potential role of OP-1 to overcome pseudarthrosis. Spine 2004;29: 1405-12.

8. Palumbo $M$, Valdes $M$, Robertson A, Sheikh $\mathrm{S}$, Lucas P. Posterolateral intertransverse lumbar arthrodesis in the New Zealand White rabbit model: I. Surgical anatomy. Spine J 2004;4:287-92.

9. Valdes $M$, Palumbo M, Appel AJ, McAllis ter S, Ehrlich M. Posterolateral in tertransverse lumbar arthrodesis in the New Zealand white rabbit model: II. Operative technique. Spine J 2004;4:2939.

10. Cheah PS, Low CK, Mohidin N, et al. Ket amine-xylazine/tiletamine-zolazepam pro longed anesthesia in Cyanomolgus mon keys. Online Journal of Veterinary Research 2007;11:39-45.

11. Boden SD, Moskovitz PA, Morone MA, Toribitake Y. Video-assisted lateral intertransverse process arthrodesis. Validation of a new minimally invasive lumbar spinal fusion tecnique in the rab bit and nonhuman primate (rhesus) mod els. Spine 1996;21: 2689-97. 